\title{
ENERGY MANAGEMENT FOR DEVICES SUPPORTING DEMENTIA PATIENTS
}

\author{
Diogo Silva, Fernando Luís-Ferreira, João Sarraipa and Ricardo Gonçalves \\ CTS, UNINOVA, Dep. ${ }^{o}$ de Eng. ${ }^{a}$ Electrotécnica Faculdade de Ciências e Tecnologia, Universidade Nova de Lisboa \\ 2825-529 Caparica, Portugal
}

\begin{abstract}
The advances in medicine and the improvements in healthcare have provided treatment and cure for many pathologies and awarded a larger expectancy of life. It represents a considerable improvement in ensuring treatment for diseases otherwise mortal and thus becoming, in some cases, chronic disorders that a person can live with for many years. This evolution has a tremendous impact in the overall healthcare environment and thus promoting longer life expectancy especially in developed countries. It is necessary, however, to consider that by increasing the width of the top level of the aging pyramid, it is therefore expected an increased incidence of the ageing related disorders. In that matter, the raising prevalence of Dementia cases is noticeable and is among the most prominent worries for the health community, as expressed in the World Health Organization reports. It is therefore of major relevance to provide assistance to citizens with dementia, especially in what concerns with losing cognitive functions such as the notion of self, awareness of current location and identifying the need to return home or to known places. The present research work is bases on the usage of smartphones to track people and has a specific target, in this matter, in extending battery life and for that matter the time a person can be located and assisted. The strategy, applicable to smartphones or other devices, relies on ensuring that energy is wisely managed so that batteries have an extended duration, increasing the chances that devices are still connected and accessible when most needed, such as while a person is experiencing a wandering episode. The aim of the present work is to focus on energy consumption to increase the chances to locate a person that is lost, with limited cognitive functions and most likely at risk for health or when even survival could be endangered.
\end{abstract}

\section{KEYWORDS}

Dementia, Wandering, Smartphone App, Energy Optimization

\section{INTRODUCTION}

Today the expectancy of life has reached levels that where unthinkable centuries ago. The major part of the population in developed countries have access to healthcare that allows prevention and treatment for most of the pathologies once considered deadly, sooner or later. It is thus expectable that with the extended lifetime the prevalence of aging associated pathologies becomes more problematic for people and the society. Dementia, in its various forms is a major problem recognized by the World Health Organization as a public health priority. Dementia is becoming a problem for healthcare systems that will become worse with time as people's lives will become longer. The benefit of better healthcare is putting a burden on the quality of life for those living longer and a challenge to support healthy longer life. Dementia is a syndrome that affects memory, thinking, behavior and ability to perform everyday activities. The number of people living with dementia worldwide is currently estimated at 35.6 million. This number will double by 2030 and more than triple by 2050 (Alzheimer's Disease International, 2012). There are several types of dementia, depending on the causes and symptoms but the consequences are rather similar and the problems they rise are almost the same. Alzheimer is the most prevalent with about 60 to $70 \%$ of the cases. Then it comes Vascular Dementia as the second most frequent cause for dementia, about $15 \%$ of the cases but, unlike Alzheimer, without any licensed treatment (O'Brien and Thomas, 2015), The other types are front temporal dementia, Dementia of Lewy corps which is a neurodegenerative disorder resulting in slowly progressive and unrelenting dementia until death (Weisman and McKeith, 2007) and the well-known Parkinson's disease. Those types of dementia share common characteristics that emerge from cognitive impairments. It is likely that patients in different stages present different symptoms and that, as time passes, the patterns and activity level of the disease are 
changing, in most of the cases getting worse over time. The common behaviors that worry carers and families are the tendency to forget basic information, such as name and address and, for that reason, the tendency to wander without known destination as the person forgets what was doing or where is home. Those are risky situations that can be addressed by devices that a person can carry and help in getting their location.

\section{ANALYSIS OF ENERGY CONSUMPTION}

Currently, energy consumption is a hotly contested topic all over the world. The objective is to find effective methods to extend the life of the devices in each load. Patients with dementia are no exception and, due to the problems in remembering paths and places, it is important that they can be located. For that it is necessary that devices have tracking information and, more important than that, devices must be working. For these patients that need daily follow-up due to lack of orientation it is important that unless they are accompanied, it is possible to reach them by any available device. In order to keep their independence and continue to become integrated in society, it is better to use electronic devices that most people use in daily routine and, at the same time, keep them trackable and connected to the caregivers. The easiest devices to find by the end consumer that meet these requirements are smartphones. For this reason, it's decisive to optimize resources for a better battery management. That is the aim of the current work.

In previous works (Luis-Ferreira et al., 2018) research has been focused in the devices and applications that can provide location and help the patients with the adequate features to detect risks and provide timely communication of detected risks and location, to carers and family. The problems then identified were related with the extension of the absence of notice and the time a person can be lost by wandering in unknown places. That significantly arises the question that, when the person most need it is possible that the devices are shut down by lack of energy.

A reasonable approach to address this problem is to manage, optimized through machine learning, the usage of sensors and antennas in smartphones. In our research study, as observed in the following graphs, energy consumption of the sensors and antennas may vary in different situations.

The consumption patterns will take in account not only the devices but also the applications being used and the result, in terms of consumption of both software applications and hardware devices being used for the same objective. It was noticed a dramatic increase in energy consumption once the smartphone actually starts transmitting data over the Wi-Fi antenna. The value climbs almost three times if we run an application such as Google Maps, which uses GPS and Wi-Fi simultaneously, while rendering images dynamically (Corral et al., 2013). In Figure 1 it is possible to observe our early basis of work by observing the different consumption patterns for devices in a smartphone (Nokia N95) that gives clues about where energy is spent and what benefits can be obtained with a proper management of those devices (Perrucci, Fitzek and Widmer, 2011).

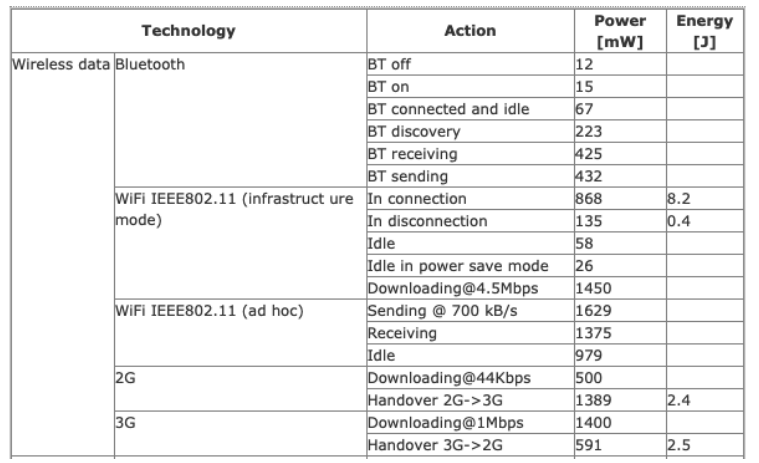

Figure 1. Energy Consumption in smartphone communication devices

It is observed that the use of GPS causes major losses of battery when compared with other sensors like accelerometers that are known to provide guidance for short periods or time or small to medium displacements by evaluating relative displacements. So, it is deductible that its usage should be kept to strictly necessary. It is still interesting to note that GPS, although more precise on the outside, consumes more energy in this situation. There are still other antennas that help locating the device such as the Wi-Fi antenna. Figure 2 presents the different energy consumption patterns for a user moving and standing either inside or outside a building. 


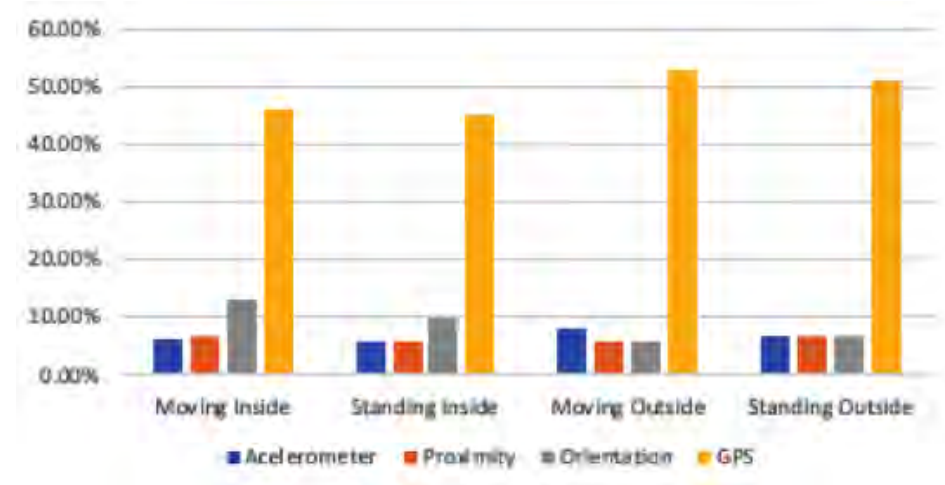

Figure 2. Energy Consumption with data transmission

In the previous chart, it becomes evident that the Wi-Fi, GSM and Bluetooth antennas consume less energy than GPS. For this reason, they can be used, when near to other recognizable devices, to locate the patient's device it can thus be applied to smartwatches that can use the smartphone location devices. Another important strategy to be applied in these cases is the intermittent activation of the GPS that can minimize the impact of that device in energy consumption patterns and still ensure that location is stored and provided from time to time. Table 1 presents the usage of different devices according to the present situation, making possible to extend the battery life by switching on and off the sensors that are not needed in the present moment. The different devices in a smartphone have different consumption levels meaning different weight in the overall consumption of a device's battery. The consumption patterns also vary according to the activity a person is executing while indoors or outside and such results can be observed next in Table 1 (Inayat Khan et al, 2016).

Table 1. Sensors relative Energy consumption

\begin{tabular}{|c|c|c|c|c|}
\hline Sensor & $\begin{array}{c}\text { Interior } \\
\text { Movement }\end{array}$ & $\begin{array}{c}\text { Standing } \\
\text { Inside }\end{array}$ & $\begin{array}{c}\text { External } \\
\text { Movement }\end{array}$ & $\begin{array}{c}\text { Standing } \\
\text { Outside }\end{array}$ \\
\hline Accelerometer & $6,35 \%$ & $5,95 \%$ & $8 \%$ & $6,6 \%$ \\
\hline Proximity & $6,7 \%$ & $5,9 \%$ & $5,9 \%$ & $6,9 \%$ \\
\hline Orientation & $13,23 \%$ & $9,7 \%$ & $5,9 \%$ & $6,9 \%$ \\
\hline GPS & $46 \%$ & $45,22 \%$ & $53 \%$ & $51 \%$ \\
\hline
\end{tabular}

Beyond comparison, there are different profiles of consumption according to behavioural status. This is the case, for the same person, that moves, goes outside or returns inside buildings. Those patterns as observed next in Figure 3.

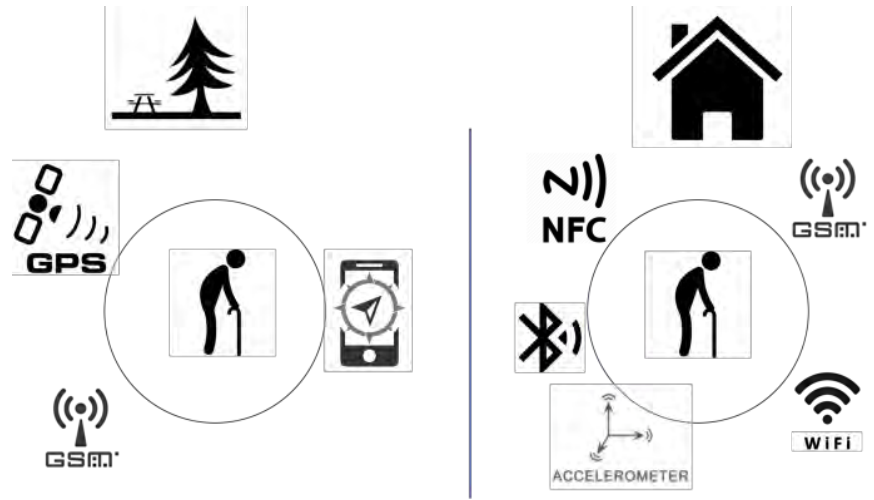

Figure 3. Devices selected according to location

The energy management information becomes of major importance by allowing an optimization of resources in smartphones for people who are in situations that need constant monitoring. The present work comes as a continuation of the early work on the development of personalized technological architectures to 
assist dementia patients based on energy consumption profiles which become essential for the successful implementation of the current work where those profiles are in fact brought to implementation (Luis-Ferreira et al., 2019).

\section{CONCLUSION}

The presented research is being carried in the scope of an ongoing MSc in Electronics and Computer Science and is applied in the research work for the CARELINK AAL project. Work carried so far passed early stages as it was verified the high potential of energy management strategies to extend the autonomy of mobile devices. This smart management of energy is of most importance for patients with Dementia, as sometimes, cognitive problems appear without notice in episodes where the person manifests lack of orientation, lost sense of time and place and in general inability to return home or to a known place. In these cases, people also tend to distance themselves from a non-controllable situation and refuse to admit their situation and thus are not likely to ask for help even while carrying a communications device. In those cases, it becomes important to have an active device to make possible location and contact, to make possible reaching this people while in a situation that can be of risk or even life threatening. It is known that the first 72 hours that a person is lost are critical, with time passing, without any option to locate them time runs against the possibilities of a good outcome. The most common and used solutions for communication and location services are in fact the smartphones but other customized dispositive can also be used. The study hereby presented addresses the need to extend, as much as possible, the autonomy of the devices which, when dry out of energy, become completely useless for tracking and rescue. From the current tests it Is possible to envisage an extension of battery above $20 \%$ making a contributing factor to promote the possibility that lost people can be found and become safe before it is too late.

\section{ACKNOWLEDGEMENT}

The authors acknowledge the European Commission for its support and partial funding and the partners of the research project CARELINK, AAL-CALL-2016- 049 funded by AAL JP, and co-funded by the European Commission and National Funding Authorities of Ireland, Belgium, Portugal and Switzerland.

\section{REFERENCES}

Alzheimer's Disease International, W. H. O. (2012) Dementia : A Public Health Priority. World Health Organization.

Corral, L. et al. (2013) 'A method for characterizing energy consumption in Android smartphones', in 2013 2nd International Workshop on Green and Sustainable Software (GREENS). IEEE, pp. 38-45. doi: 10.1109/GREENS.2013.6606420.

Inayat Khan et al (2016) 'Sensors are Power Hungry: An Investigation of Smartphone Sensors Impact on Battery Power from Lifelogging Perspective', Bahria University Journal of Information \& Communication Technologies, 9(2), pp. 8-19. Available at: https://www.bahria.edu.pk/ojs/index.php/bujict/article/view/52 (Accessed: 28 February 2019).

Luis-Ferreira, F. et al. (2018) 'Assessment of Risk for Alzheimer Patients by Monitoring Heartbeat and Identifying Falls', in Proceedings of 10th International Conference on e-Health 2018. Madrid, Spain.

Luis-Ferreira, F. et al. (2019) 'Personalised Technological Architectures to Assist Dementia Patients based on Energy Efficiency', in ICIST 2019 - 9th International Conference on Information Society and Technology. Kopaonik, Serbia.

O'Brien, J. T. and Thomas, A. (2015) 'Vascular dementia', The Lancet. Elsevier, 386(10004), pp. 1698-1706. doi: 10.1016/S0140-6736(15)00463-8.

Perrucci, G. P., Fitzek, F. H. P. and Widmer, J. (2011) 'Survey on Energy Consumption Entities on the Smartphone Platform', in 2011 IEEE 73rd Vehicular Technology Conference (VTC Spring). IEEE, pp. 1-6. doi: 10.1109/VETECS.2011.5956528.

Weisman, D. and McKeith, I. (2007) 'Dementia with Lewy Bodies', Seminars in Neurology. Copyright (C) 2007 by Thieme Medical Publishers, Inc., 333 Seventh Avenue, New York, NY 10001, USA., 27(1), pp. 042-047. doi: 10.1055/s-2006-956754. 